\title{
Prozessvertrauen als Forschungsverfahren, empirische Erkenntnis als Gabe: qualitative Soziologie im Licht hermeneutisch- theologischer Ansätze
}

Barbara Grimpe

Die Soziologie bietet eine breite Palette an Forschungsliteratur über Vertrauen, und insofern macht es Sinn, sie in ein interdisziplinäres Vertrauensprojekt einzubinden. Nachfolgend gebe ich zunächst Beispiele für eine soziologische Vertrauensbetrachtung. Dabei konzentriere ich mich auf die in empirischer Hinsicht qualitative Soziologie und skizziere nur einige Forschungslinien. Darauf bauen meine weiteren Überlegungen zu Interdisziplinarität auf, wobei ich mich auf das Verhältnis zur Theologie und auch hier nur auf ausgewählte Aspekte beschränke. Es ist ein dynamisches Verhältnis: Zunächst gehe ich auf den vermutlich unvermeidlichen, aber nicht tragischen Abschied von Interdisziplinarität angesichts lauter Innerdisziplinaritäten ein - danach ausführlicher auf >Prozessvertrauen und >Vertrauen als Gabe` als zwei Konzepte bzw. Orte, wo sich die qualitative Soziologie und hermeneutische Theologie unerwartet wieder getroffen haben.

Untersucht man beispielsweise Mikrokredite und genauer die historisch-kulturelle Transformation des Mikrofinanzsektors der sog. Entwicklungs- und Schwellenländer in einen globalen Markt, so bietet sich schon vor irgendeiner spezifischen empirischen Auseinandersetzung eine etablierte wirtschaftssoziologische Grundinterpretation an: Kreditbeziehungen können als doppelte Vertrauensbeziehungen in dem Sinn verstanden werden, dass zum einen Gläubiger wie Schuldner über die Dauer der Darlehensvergabe und sukzessiven Rückzahlung hinweg, also für die Dauer ihres sintertemporalen Austauschs`, auf den bleibenden Wert des Geldes vertrauen müssen (Vertrauen in Geld als Institution); zum anderen sieht sich der Gläubiger mit einem spezifischeren Vertrauensproblem konfrontiert, denn er befindet sich gegenüber der jeweiligen Kreditnehmerin oder dem Kreditnehmer in einer Situation der »asymmetrischen Information«: "Wird sie [oder er] den Kredit in einem Jahr zurückzahlen? « ${ }^{1}$

\footnotetext{
1 Bruce G. Carruthers, The Sociology of Money and Credit, in: Handbook of Economic Sociology, hg. v. Neil J. Smelser/Richard Swedberg, Princeton 2005, 355-378, hier: 362-363; Übersetzung aus dem Englischen:BG).
} 
Schaut man nun empirischer etwas genauer auf den gegenwärtigen Mikrofinanzsektor und führt etwa eine Diskursanalyse der offiziellen Publikationen von internationalen Entwicklungsorganisationen und auf Mikrofinanzierung spezialisierten Mikrofinanzfonds durch, so zeigt sich beispielsweise, dass für diesen intertemporalen Austausch nicht nur finanzielle Gewinne (für den Gläubiger), sondern auch ssoziale Gewinne für den Kreditnehmer, etwa ein Beitrag zur Armutsminderung, in Aussicht gestellt werden. ${ }^{2}$ Dieses doppelte Gewinnversprechen impliziert weitere Vertrauensbegriffe. Denn um die sozialen Gewinne nachzuweisen, bedienen sich viele Fonds wie öffentliche Entwicklungsorganisationen einer Vielzahl von zum Teil durch komplexe Erhebungsverfahren generierten Kennzahlen (z.B. der >Progress out of Poverty Index; Grameen Foundation 2008). Aus Sicht der Soziologie der Quantifizierung lässt sich angesichts dieser umfangreichen, in den Publikationen der Akteure selbst nur geringfügig hinterfragten Berechnungsarbeiten darauf schliessen, dass die Finanz- und Entwicklungsexperten des Feldes grosses Vertrauen in Zahlen, Kalkulationen und allgemeiner Systemvertrauen haben bzw. solches bei ihrem Publikum, den potentiellen Investoren, voraussetzen. ${ }^{3}$

Schaut man nun empirisch noch etwas genauer hin, indem man z.B. einige der Finanz- und Entwicklungsexperten zum qualitativen Interview bittet, stellt sich heraus, dass diese 'Zahlenmenschen<, die den Publikationen nach also grosses Vertrauen in Rechenwerke zu setzen bzw. dieses vorauszusetzen scheinen, genauso als ‘Zahlenskeptiker $z u$ bezeichnen sind. ${ }^{4}$ Was die Diskursanalyse erbrachte, ist nur die eine Seite der Medaille: Das Vertrauen in Zahlen wird durch

2 CGAP 2011, Foreign Capital Investment in Microfinance. Reassessing Financial and Social Returns: http://www.cgap.org/gm/document-1.9.2584/FN44.pdf (August 31, 2012); vgl. World Bank 2013, ’News \& Broadcast - Microfinance and Financial Inclusions: http://web.worldbank.org/WBSITE/EXTERNAL/NEWS/0, contentMDK:20433592 menuPK:34480 pagePK:64257043 piPK:437376 theSite PK:4607,00.html (Januar 28, 2013).

3 Wendy Espeland/Mitchell Stevens, A Sociology of Quantification, in: Arch. europ. sociol. 49/3 (2008), 401-436; Andrea Mennicken, Figuring Trust: The Social Organization of Credit Relations, in: Facts and Figures: Economic Representations and Practices, Ökonomie und Gesellschaft, hg. v. Herbert Kalthoff/Richard Rottenburg/ Hans-Jürgen Wagener, Marburg 2000, 35-58; Niklas Luhmann,Vertrauen. Ein Mechanismus der Reduktion sozialer Komplexität, Stuttgart 1968; vgl. Theodore Porter, Trust in Numbers: the Pursuit of Objectivity in Science and Public Life, Princeton 1995.

${ }^{4}$ Uwe Vormbusch, Zahlenmenschen als Zahlenskeptiker. Daten und Modelle im Portfoliomanagement, in: Soziologie der Finanzmärkte, hg. v. Herbert Kalthoff/Uwe Vormbusch, Bielefeld, 2012, 313-337. 
persönliches Vertrauen (und Misstrauen) geprüft und ausbalanciert. So lautet etwa eines der konkreten Forschungsergebnisse: Müssen sich Fondsmanager dafür entscheiden, mit einer Mikrofinanzinstitution eine längerfristige Geschäftsbeziehung einzugehen, so begeben sie sich an Ort und Stelle und führen mit verschiedenen Mitarbeitern dieser Organisation Gespräche, bei denen es immer wieder auf das interaktiv und situativ, oft stumm und verkörpert gewonnene "Gefühl« ankommt (wie sich mehrere Experten ausdrückten), ob eine Investition wohl tatsächlich in Zukunft einen doppelten - finanziellen wie sozialen - Gewinn bringt. In theoretischer Hinsicht bedeutet dies u.a. eine Verschiebung von der Soziologie der Quantifizierung zur Ethnomethodologie. Nun stellt sich z.B. die Frage, welche Merkmale die konkrete Interaktion zwischen dem Fondsvertreter und dem Mitarbeiter vor Ort aufwies, so dass erstgenannter Vertrauen fassen konnte, oder auch nicht. ${ }^{5}$

Über diese drei Zugangsweisen - von der wenig empirischen wirtschaftsoziologischen Ausgangsbetrachtung über die diskursanalytisch relevant gewordene Soziologie der Quantifizierung und die per qualitatives Interview wiederum hervorgetretene Ethnomethodologie - haben sich nicht nur verschiedene Vertrauensaspekte gezeigt. Es hat sich auch das Gesicht `der Soziologie immer wieder verändert. Dabei war bis jetzt nur von wenigen qualitativen Forschungsansätzen die Rede und noch nicht einmal von der breiten quantitativen Sozialforschung. Für die interdisziplinäre Zusammenarbeit, etwa mit der Theologie, aber genauso auch mit der (Neuro-)Ökonomie, Psychologie und Geschichte heisst dies: Die Soziologie tritt den anderen kaum als einheitliche Disziplin mit einfach bestimmbaren Vertrauenskonzepten gegenüber. Umgekehrt haben auch die anderen Disziplinen so viele Gesichter, dass eine als sinterdisziplinärı angelegte Vertrauensforschung in der praktischen Umsetzung zunächst einmal ein langwieriges wechselseitiges Entdeckungsverfahren ist, bei dem Verwirrung und wechselseitiges Nichtverstehen dominieren. Da das Universitätssystem insgesamt aber weiterhin disziplinär ausgerichtet ist, muss sich meines Erachtens jede an einem interdisziplinären Projekt beteiligte Fachrichtung den Grossteil der Zeit mit der eigenen sinneren (Vielstimmigkeit auseinandersetzen (um das eigene spezifische Projekt darin zu verorten) und kann erst im zweiten Schritt versuchen, dieses eigentliche Stimmengewirr auch im Verhältnis zu den anderen Fachrichtungen zu sortieren. Der dritte, sehr unwahrscheinliche Schritt besteht in der Identifizierung tatsächlich gemeinsamer Forschungslinien.

5 Harold Garfinkel, Studies in Ethnomethodology, Englewood Cliffs 1967, 172-173. 
Zwei Beispiele für solche gemeinsamen Forschungslinien sind aus meiner Sicht die Konzepte des Prozessvertrauens und von Vertrauen als Gabe. Dass hier eine Überschneidung zwischen qualitativer Soziologie und hermeneutischer (sowie teils praktischer, teils philosophischer) Theologie besteht, war meiner Wahrnehmung nach für die Beteiligten im Voraus nicht absehbar, sondern ist selbst Prozessergebnis und Gabe, oder anders gesagt, ein Beispiel für Wissenschaft als "Mangle of Practice« ${ }^{6}$

Ingolf U. Dalferth und Simon Peng-Keller bezeichnen "Prozessvertrauen ${ }^{7}$ als eine bisher wenig erforschte Form von Vertrauen, die in »ergebnisoffenen Formen von Kommunikation" und konkret u.a. in der praktischen beruflichen Tätigkeit von Psychotherapeuten, Pädagogen und Seelsorgern eine wichtige Rolle spiele. Sie nennen eine Reihe von Merkmalen dieses Vertrauenstyps: die betreffende Person (Psychotherapeut, Pädagoge, Seelsorger) müsse bereit sein, "Nicht-Verstehen" und gar längere "Phasen der Unklarheit und Ungewissheit " gegenüber dem Gesprächspartner (Patient, Kind usw.) auszuhalten. "Vorannahmen, Deutungen und Ziele» seien "vorübergehend einzuklammern, um sich besser dem aktuellen [...] Geschehen öffnen zu können«. Denn es würden sich erst in einem "unüberschaubaren und teilweise chaotisch wirkenden Suchprozess Lösungsmöglichkeiten entwickeln, die anders nicht zu finden sind «. ${ }^{8}$ Wenn es nun dem Therapeuten, Pädagogen und Seelsorger paradoxerweise gelingt, auf diese so gar nicht absehbaren Lösungsmöglichkeiten zu vertrauen, so lässt sich dies den beiden Autoren zufolge als "Prozessvertrauen" bezeichnen. Um aber nicht in der eigenen Angst ob dieser Unvorhersehbarkeit zu versinken und im Gesprächsprozess präsent zu bleiben, bedürfe es, so Dalferth und Peng-Keller in Anlehnung an Elizabeth Strand ${ }^{9}$ weiter, »eine[r] genauen[n] und differenzierte[n] Fremd- und Selbstwahrnehmung«. Erstens müsse man in der Lage sein, fremdes Verhalten waus der Beobachterperspektive" wahrzunehmen, ohne zweitens "die achtsame Wahrnehmung des eigenen Gesprächsverhaltens« zu vernachlässigen, und ohne sich drittens "der eigenen Emotionalität« zu verschliessen. ${ }^{10}$

\footnotetext{
6 Andrew Pickering, The Mangle of Practice: Agency and Emergence in the Sociology of Science, in: The American Journal of Sociology 99/3 (1993), 559-589.

7 Ingolf U. Dalferth/Simon Peng-Keller, (Hg.) Kommunikation des Vertrauens, Leipzig 2012, 205-208.

8 Ebd., Hervorhebungen B.G.

9 Elizabeth Strand, Non-Anxious Presence. A Key Attribute of the Successful Veterinarian, in: Journal of Veterinary Medical Education 33/1 (2006), 65-70.

${ }^{10}$ Dalferth/Peng-Keller (Hg.), Kommunikation des Vertrauens, 208.
} 
Es gibt also physisch und psychisch viel zu tun für die Therapeutin, den Pädagogen und die Seelsorgerin, wenn sie diejenigen Lösungsmöglichkeiten, »die anders nicht zu finden sind « erschliessen wollen. Und dass sich diese in der Tat auftun, ist bei all dem Aufwand trotzdem nicht gesagt.

Meines Erachtens macht dieses buchstäblich unabsehbare Prozessvertrauen den Kern der - zugegebenermassen äussersten Formen der qualitativen empirischen Forschung in der Soziologie aus. Dalferths und Peng-Kellers Beobachtungen stehen, auch wenn sich die beiden mit einem eigenen "hermeneutisch-phänomenologische[n] Zugang" explizit gegen »empirische Studien, die auf methodischer Datenerhebung und -auswertung beruhen", darunter namentlich die teilnehmende Beobachtung, abgrenzen wollen, ${ }^{11}$ der reflexiven Literatur über die Ethnographie als Erforschungsweise des Fremden und Eigenen sehr nahe (z.B. Berg/Fuchs 1993 ${ }^{12}$ ). Dass sich die beiden Autoren aber so deutlich gegen "empirische Studien" abgrenzen, könnte mit einem bestimmten Empirieverständnis zusammenhängen, welches sich in folgender Formulierung andeutet: Eine "Hermeneutik des Vertrauens" ziele auf eine andere "Art der Beteiligung" ab, indem sie zu beschreiben versuche, "was wir tun, wenn wir vertrauen, nicht vertrauen oder misstrauen $\ll .{ }^{13}$ Vielleicht wird hier angenommen, die qualitativen Soziologen würden die Verwicklung der eigenen Perspektiven, Identität und Hoffnungen (auf interessante Ergebnisse) im Forschungsverlauf, in diesem Sinn das ,Wir der Forscher, ausblenden, und an einer starren Unterscheidung zwischen >Gegenstand Dies trifft aber gerade nicht für die ethnographische teilnehmende Beobachtung und verwandte Forschungsformen, etwa das ethnographische Interview, zu (vgl. Spradley 19794). Es ist anerkanntermassen Chance und Schwäche zugleich, dass Ethnograph und Ethnographin buchstäblich selbst die Forschungsinstrumente sind, durch die hindurch sich die Felderfahrungen sinnlich ereignen. ${ }^{15}$ Es ist eine genauso schmerzhafte wie notwendige und schliesslich auch wieder bereichernde komplexe Aufgabe, als Ethnograph immer

\footnotetext{
11 Ebd., 197.

12 Eberhard Berg/Martin Fuchs, Hg., Kultur, soziale Praxis, Text: die Krise der ethnographischen Repräsentation, Frankfurt a.M. 1993.

${ }^{13}$ Dalferth/Peng-Keller (Hg.), Kommunikation des Vertrrauens, 197; Hervorhebungen im Original.

${ }^{14}$ Vgl. James Spradley, The Ethnographic Interview, New York 1979.

15 Stefan Hirschauer, Ethnografisches Schreiben und die Schweigsamkeit des Sozialen. Zu einer Methodologie der Beschreibung, in: Zeitschrift für Soziologie 30/6 (2001), 429-451.
} 
wieder neu die eigenen Vorannahmen und Vorstellungen zu erkennen und sie ggf. zu verwerfen. Das Gehörte und Gesagte ist ordnend zu interpretieren, während dem Beobachteten oder Gesprächspartner dauernd grösstmögliche Aufmerksamkeit entgegenzubringen ist, um seine Sicht (nicht zu wiederholen sondern) analytisch dicht zu rekonstruieren. In der Folge stellt dieses Verfahren oft (dummerweise!) die eben zurechtgelegten Interpretationen wieder in Frage. Insgesamt wird in diesem Fremd- und Selbsterforschungsprozess idealerweise auch das o.g. / Wir genauer bestimmt. Worauf am Ende dieses oft holperigen Arbeitswegs zu hoffen ist, sind empirische und letztlich auch theoretische Erkenntnisse, die, so die Annahme, durch keine andere Zugangsweise zu finden sind.

Ich erlaube mir, mit etwas Übertreibung, diese Erkenntnisse im theologischen Sinn als Gabe zu bezeichnen. Natürlich kann ich diese theologische Perspektive, wie ich sie im Austausch mit Marcel Egli und in Bezug auf sein Dissertationsprojekt zu verstehen versuchte, 16 nachfolgend nur sehr rudimentär und durch meine eigene Wahrnehmung überformt wiedergeben. Es geht mir um das Konzept von Gottvertrauen bzw. Gottesglaube als Gabe (wobei eigentlich schon Glaube und Vertrauen theologisch aufwändig zu unterscheiden wären). Demnach lässt sich Gottesglaube und -vertrauen im Menschen nicht planvoll und kontrolliert einsetzen und aufbauen; beides liegt ausserhalb jeglicher menschlicher Einflussnahme und widerfährt dem Menschen. (In dieser /Widerfahrnis wird sogar der Mensch erst Mensch, d.h. das Menschliche kann sogar gar nicht getrennt von der Gotteserfahrung gedacht werden.) Die Erfahrung von Gottesglaube und -vertrauen sind unverfügbar, sie ereignen sich, sie brechen irgendwann über einen herein, oder eben auch nicht (und vielleicht könnte man ergänzen, dass auch Gotteszweifel und -misstrauen entsprechend den noch so Gläubigen unerwartet ereilen und quälen können). Es handelt sich um Gaben in dem Sinn, dass tatsächlich Gott und kein anderer Vertrauen und Glauben eingibt oder nicht. So müsste man z.B. sprachlich verwirrend, aber inhaltlich womöglich zutreffender das eigene Gottvertrauen nicht als ein sich vertraue Gott<, sondern `Es wird mir immer schon vertraut « (durch Gott, vorgängig) ausformuliert werden. Auch in der forschenden Haltung, d.h. als wissenschaftlicher Beobachter, kann der Untersuchungsgegenstand des Gottesglaubens und -vertrauens nur nachgedacht werden, im Wortsinn: Es ist auch hier wieder Gott, der mit seinem Handeln dem Forschungshandeln und -denken vorgängig

\footnotetext{
${ }^{16}$ Marcel Egli, Vertrauen und geschenkte Identität. Vertrauen in theologischer Perspektive im Anschluss an die Versöhnungslehre Karl Barths (Arbeitstitel).
} 
ist und in der theologischen Analyse nicht mehr als nachträglich gedacht werden kann.

In der Soziologie gibt es Begriffe wie sunintendierte Effekter und 'Emergenz`, mit denen in einem vielleicht weniger existenziellen Sinn, aber doch inhaltlich verwandt auf die Unverfügbarkeit von sozialer Ordnung aufmerksam gemacht werden soll. Und auch der qualitative Forschungsprozess selbst birgt im Idealfall empirische und theoretische Erkenntnisse, die tatsächlich nicht absehbar sind, dem Forschenden widerfahren können - oder allzuoft auch nicht. Zudem gibt es die breitere Bewegung des Konstruktivismus, die auf vielfalltige Weise auf die (perspektivische) Begrenztheit und Vorläufigkeit von Forschungsergebnissen aufmerksam macht. Meines Erachtens lohnt sich zur Stärkung dieser existierenden soziologischen (und allgemeineren) Denklinien und Begriffe ein Exkurs in die vorher nur skizzenhaft wiedergegebene, aber äusserst eindrückliche 'Methodologier der Theologie, wie ich sie hier jetzt einmal salopp nenne. Denn denkt man lebensweltliche und Forschungsprozesse so radikal immer von Gott her und durch ihn hindurch (und, wie eben erläutert, sowieso nur nachträglich), so heisst das möglicherweise nichts anderes, als dass die Theologie in grösstmöglichem Ausmass die Begrenztheit menschlichen Verstehens und Wissens in Rechnung stellt.

Tröstlich für die Forscherseele und auch einfach schön zu lesen sind in dieser Hinsicht Max Webers Überlegungen zum »Einfall» in seinem Aufsatz Wissenschaft als Beruf, den er vor fast einhundert Jahren geschrieben hat. Man kann sich Webers Auffassung, dass die Plackerei so mancher Forschungstätigkeit und die "Leidenschaft" zu forschen zwar keine Garantie für gute Einfälle sind, diese aber immerhin »locken " können, hoffnungsvoll anschliessen:

"Nur auf dem Boden ganz harter Arbeit bereitet sich normalerweise der Einfall vor. Gewiß: nicht immer. [...] Der Einfall ersetzt nicht die Arbeit. Und die Arbeit ihrerseits kann den Einfall nicht ersetzen oder erzwingen, so wenig wie die Leidenschaft es tut. Beide - vor allem: beide zusammen - locken ihn. Aber er kommt, wenn es ihm, nicht, wenn es uns beliebt «. ${ }^{17}$

\footnotetext{
17 Max Weber, Wissenschaft als Beruf, in: ders., Schriften 1894-1922, hg. v. Dirk Kaesler, Stuttgart 1919, 474-511; Hervorhebungen im Original.
} 


\section{Literatur}

Berg, Eberhard/Martin Fuchs, Hg., Kultur, soziale Praxis, Text: die Krise der ethnographischen Repräsentation, Frankfurt a.M. 1993.

Carruthers, Bruce G., The Sociology of Money and Credit, in: The Handbook of Economic Sociology, hg. v. Neil J. Smelser/Richard Swedberg, Princeton 2005, 355-378.

CGAP, Foreign Capital Investment in Microfinance. Reassessing Financial and Social Returns, 2011: http://www.cgap.org/gm/ document-1.9.2584/FN44.pdf (August 31, 2012).

Dalferth, Ingolf/Simon Peng-Keller, Vertrauenskommunikation in professionellen Kontexten. Diskussionsstand und Forschungsperspektiven aus hermeneutischer Sicht, in: Kommunikation des Vertrauens, hg. v. Ingolf U. Dalferth/Simon Peng-Keller, Leipzig 2012, 195-221.

Espeland, Wendy/Mitchell Stevens, A Sociology of Quantification, in: Arch. europ. sociol. 49/3 (2008), 401-436.

Garfinkel, Harold, Studies in Ethnomethodology, Englewood Cliffs 1967.

Grameen Foundation, Progress out of Poverty Index. PPI Pilot Training. Participant Guide, 2008: http://www.progressoutofpoverty. $\mathrm{org} /$ system/files/PPITrainingGuide.pdf (August 31, 2012).

Hirschauer, Stefan, Ethnografisches Schreiben und die Schweigsamkeit des Sozialen. Zu einer Methodologie der Beschreibung, in: Zeitschrift für Soziologie 30/6 (2001), 429-451.

Luhmann, Niklas, Vertrauen. Ein Mechanismus der Reduktion sozialer Komplexität, Stuttgart 1968.

Mennicken, Andrea, Figuring Trust: The Social Organization of Credit Relations, in: Facts and Figures: Economic Representations and Practices, Ökonomie und Gesellschaft, hg. v. Herbert Kalthoff/Richard Rottenburg/Hans-Jürgen Wagener, Marburg, 2000, 35-58.

Pickering, Andrew, The Mangle of Practice:Agency and Emergence in the Sociology of Science, in: The American Journal of Sociology 99/3 (1993), 559-589.

Porter, Theodore, Trust in Numbers: the Pursuit of Objectivity in Science and Public Life, Princeton 1995.

Spradley, James. 1979. The Ethnographic Interview, New York 1979. Strand, Elizabeth, Non-Anxious Presence. A Key Attribute of the Successful Veterinarian, in: Journal of Veterinary Medical Education 33/1 (2006), 65-70.

Vormbusch, Uwe, Zahlenmenschen als Zahlenskeptiker. Daten und Modelle im Portfoliomanagement, in: Soziologie der Finanz- 
märkte, hg. v. Herbert Kalthoff/Uwe Vormbusch, Bielefeld, 2012, 313-337.

Weber, Max, Wissenschaft als Beruf, in: ders., Schriften 1894-1922, hg. v. Dirk Kaesler, Stuttgart 1919, 474-511.

World Bank, News \& Broadcast - Microfinance and Financial Inclusion, 2013: http://web.worldbank.org/WBSITE/EXTERNAL/ NEWS/0,,contentMDK:20433592 menuPK:34480 pagePK: 64257043 piPK:437376 theSitePK:4607,00.html (January 28, 2013 .

- Dr. Barbara Grimpe, Soziologin, hat im Rahmen des Projekts «Vertrauen verstehen> zum Thema ,Die Expansion von Mikrofinanzen aus den ökonomischen Nischen der Entwicklungszusammenarbeit der 1970er-Jahre in die heutigeWall Street Finance، geforscht. 